\title{
THERMOELASTIC ANALYSIS OF BENDING PROBLEMS IN FGM PLATES
}

\author{
V. SLADEK, L. SATOR \& J. SLADEK \\ Institute of Construction and Architecture, Slovak Academy of Sciences, Bratislava, Slovakia.
}

\begin{abstract}
It is well known that the original 3D elasticity problem in plate structures subjected to transversal loading can be converted to a $2 \mathrm{D}$ problem. In addition to in-plane displacements, we need to introduce the deflection and/or rotation field variables in the plate mid-plane, in order to describe displacements and deformations within the plate structure. Thus, one can develop unified formulation for bending and inplane deformation modes within the classical Kirchhoff-Love theory for bending of thin elastic plates and the shear deformation plate theories (the first order - FSDPT, and the third order - TSDPT). In this paper, we extend the derivation of the 2D formulation for coupled problems of thermoelasticity in plate structures. Three material coefficients play the role in stationary problems, namely the Young modulus, coefficient of linear thermal extension and the heat conduction coefficient. The influence of continuous gradation of these coefficients on the response of the plate subjected to thermal loadings is investigated in numerical simulations. The element-free strong formulation with using meshless approximations for spatial variation of field variables is developed.

Keywords: functional gradations of material coefficients, MLS approximations, Plate bending theories, strong formulation, thermal loading, unified formulation of $2 D$ coupled problems
\end{abstract}

\section{INTRODUCTION}

Functionally graded materials (FGM) refers to the new class of advanced composite materials utilized in modern engineering structures and frequently studied $[1,2]$ because of their superior properties. It is a well-known elimination of delamination effect in plate-like structures if the sandwich composite structures are replaced by FGM composite ones. From the physical point of view as well as from the view of engineering applications the transversal gradation of material properties is more interesting than the in-plane gradation in plate problems. The transverse gradation of Young modulus (YM) gives rise to coupling between the deflections and in-plane deformations $[3,4]$ even in the case of thin plate theory and static problems. However, it is interesting to study also the plate structures with combined gradation of material coefficients in transversal and in-plane directions because new physical effects can arise [5] in multi-graded plates.

In this paper, we shall deal with the plate problems considered within classical thermoelasticity. The unified 2D formulation is developed for FGM plates with taking into account the assumption of classical Kirchhoff-Love theory for bending of thin elastic plates as well as assumptions of the shear deformation plate theory. The material coefficients are allowed to be functionally dependent in both the transversal as well as in-plane directions. An efficient formulation is developed for numerical solution of coupled multi-field problems and some interesting effects are illustrated in numerical simulations.

\section{UNIFIED FORMULATION FOR FGM COMPOSITE PLATES UNDER THERMAL LOADING}

In the unified formulation for all three plate bending theories (KLT, FSDPT and TSDPT), the displacements can be expressed in terms of the in-plane displacements $u_{\alpha}(\mathbf{x})$, transversal displacements (deflections) $w(\mathbf{x})$ and rotations of the normal to the mid-surface $\varphi_{\alpha}(\mathbf{x})$ by 


$$
v_{i}\left(\mathbf{x}, x_{3}\right)=\delta_{i \alpha}\left\{u_{\alpha}(\mathbf{x})+\left[c_{1} \phi\left(x_{3}\right)-x_{3}\right] w_{, \alpha}(\mathbf{x})+c_{1} \phi\left(x_{3}\right) \varphi_{\alpha}(\mathbf{x})\right\}+\delta_{i 3} w(\mathbf{x})
$$

in $\mathbf{x} \in \Omega, x_{3} \in[-h / 2, h / 2]$ with $h \ll L$ where $L$ is the characteristic length in $\Omega$. Note that $i \in\{1,2,3\}, \alpha \in\{1,2\}$,

$$
\begin{gathered}
\phi\left(x_{3}\right):=x_{3}-c_{2} \psi\left(x_{3}\right), \psi\left(x_{3}\right):=4 h\left(x_{3} / h\right)^{3} / 3 \text { with } \\
c_{1}=\left\{\begin{array}{rl}
0, & \mathrm{KLT} \\
1, & \mathrm{SDPT}
\end{array}, c_{2}=\left\{\begin{array}{lr}
0, & \text { FSDPT, KLT } \\
1, & \text { TSDPT }
\end{array}\right.\right.
\end{gathered}
$$

being two key factors for switching among the three theories (KLT, FSDPT, TSDPT). Having known the dependence of displacements on $x_{3}$, we also know the dependence of strains and elastic stresses on $x_{3}$ and the original 3D thermo-elastic problem can be recast into $2 \mathrm{D}$ thermo-elastic plate bending problem. The total and thermal strains are given as

$$
\begin{gathered}
e_{\alpha \beta}(\mathbf{x}, z):=\left(v_{\alpha, \beta}+v_{\beta, \alpha}\right) / 2=\varepsilon_{\alpha \beta}(\mathbf{x})+\left[c_{1} \phi\left(x_{3}\right)-x_{3}\right] w_{, \alpha \beta}(\mathbf{x})+c_{1} \phi\left(x_{3}\right) \eta_{\alpha \beta}(\mathbf{x}) \\
\varepsilon_{\alpha \beta}=\left(u_{\alpha, \beta}+u_{\beta, \alpha}\right) / 2, \eta_{\alpha \beta}=\left(\varphi_{\alpha, \beta}+\varphi_{\beta, \alpha}\right) / 2 \\
e_{k l}^{\theta}(\mathbf{x}, z)=\alpha\left(\theta-\theta_{0}\right) \delta_{k l}
\end{gathered}
$$

and the elastic stresses in isotropic elastic plate are given as

$$
\begin{aligned}
\sigma_{\alpha \beta}\left(\mathbf{x}, x_{3}\right)= & \frac{E}{1-v^{2}} \frac{1-v}{H}\left[\tau_{\alpha \beta}^{(u)}(\mathbf{x})+c_{1} \phi\left(x_{3}\right) \tau_{\alpha \beta}^{(\varphi)}(\mathbf{x})+\left(c_{1} \phi\left(x_{3}\right)-x_{3}\right) \tau_{\alpha \beta}^{(w)}(\mathbf{x})-\right. \\
& \left.-\alpha \tau_{\alpha \beta}^{(\theta)}\left(\mathbf{x}, x_{3}\right)\right] \\
\sigma_{\alpha 3}\left(\mathbf{x}, x_{3}\right)= & \frac{E}{1+v} \frac{c_{1}}{2} \phi_{, 3}\left(x_{3}\right)\left[w_{, \alpha}(\mathbf{x})+\varphi_{\alpha}(\mathbf{x})\right] \\
\sigma_{33}\left(\mathbf{x}, x_{3}\right)= & \frac{E v}{1-v^{2}} \frac{1-v}{H}\left[u_{\gamma, \gamma}(\mathbf{x})+c_{1} \phi\left(x_{3}\right) \varphi_{\gamma, \gamma}(\mathbf{x})+\left(c_{1} \phi\left(x_{3}\right)-x_{3}\right) w_{, \gamma}(\mathbf{x})-\right. \\
& \left.-\alpha \frac{1+v}{v} \theta\left(\mathbf{x}, x_{3}\right)\right]
\end{aligned}
$$

in which $\alpha$ is the linear thermal extension coefficient, $H=1-2 v$, with $v$ and $E$ being the Poisson ratio and Young modulus (YM), respectively, and $\tau_{\alpha \beta}^{(9)}$ stand for particular strain contributions

$$
\begin{gathered}
\tau_{\alpha \beta}^{(u)}(\mathbf{x}):=H \varepsilon_{\alpha \beta}(\mathbf{x})+v \delta_{\alpha \beta} \varepsilon_{\gamma \gamma}(\mathbf{x}), \tau_{\alpha \beta}^{(\varphi)}(\mathbf{x}):=H \eta_{\alpha \beta}(\mathbf{x})+v \delta_{\alpha \beta} \eta_{\gamma \gamma}(\mathbf{x}), \\
\tau_{\alpha \beta}^{(w)}(\mathbf{x}):=H w_{, \alpha \beta}(\mathbf{x})+v \delta_{\alpha \beta} w_{, \gamma \gamma}(\mathbf{x}), \tau_{\alpha \beta}^{(\theta)}\left(\mathbf{x}, x_{3}\right):=(1+v) \theta\left(\mathbf{x}, x_{3}\right) \delta_{\alpha \beta} .
\end{gathered}
$$

In the case of FGM plate, we assume the material coefficients to be continuous functions of Cartesian coordinates as

$$
E\left(\mathbf{x}, x_{3}\right)=E_{0} E_{H}(\mathbf{x}) E_{V}\left(x_{3}\right), E_{V}\left(x_{3}\right)=1+\zeta\left(\frac{1}{2} \pm \frac{x_{3}}{h}\right)^{p}, E_{H}(\mathbf{x})=1+\kappa_{0}\left(x_{1}\right)^{b_{0}}
$$




$$
\begin{aligned}
& \alpha\left(\mathbf{x}, x_{3}\right)=\alpha_{0} \alpha_{H}(\mathbf{x}) \alpha_{V}\left(x_{3}\right), \alpha_{V}\left(x_{3}\right)=1+\xi\left(\frac{1}{2} \pm \frac{x_{3}}{h}\right)^{r}, \alpha_{H}(\mathbf{x})=1+\kappa_{1}\left(x_{1}\right)^{b_{1}} \\
& k\left(\mathbf{x}, x_{3}\right)=k_{0} k_{H}(\mathbf{x}) k_{V}\left(x_{3}\right), k_{V}\left(x_{3}\right)=1+\omega\left(\frac{1}{2} \pm \frac{x_{3}}{h}\right)^{s}, k_{H}(\mathbf{x})=1+\kappa_{2}\left(x_{1}\right)^{b_{2}}
\end{aligned}
$$

with $k$ being the heat conduction coefficient.

It is well known that the governing equations as well as the boundary conditions in unified formulation for bending problems can be derived from the principle of virtual work

$$
\delta U-\delta W_{e}=0, \delta U=\int_{\Omega}\left(\int_{-h / 2}^{h / 2} \sigma_{i j}\left(\mathbf{x}, x_{3}\right) \delta e_{i j}\left(\mathbf{x}, x_{3}\right) d x_{3}\right) d \Omega, \delta W_{e}=\int_{\Omega} p(\mathbf{x}) \delta w(\mathbf{x}) d \Omega
$$

provided that the plate is subjected to transversal loading. In order to derive the semi-integral $2 \mathrm{D}$ formulation, we need to perform the integration with respect to $x_{3}$ in closed form. Therefore, we need to know the dependence of the whole intengrand in (7) on $x_{3}$. Up to now, we presented the dependence of elastic fields on $x_{3}$ and in what follows we assume the expansion of temperature on $x_{3}$ as

$$
\theta\left(\mathbf{x}, x_{3}\right) \approx \theta_{0}+\vartheta_{0}(\mathbf{x})+z \vartheta_{1}(\mathbf{x})+z^{2} \vartheta_{2}(\mathbf{x}), z=x_{3} / h \in[-0.5,0.5]
$$

with $\vartheta_{a}(\mathbf{x})$ for $(a=0,1,2)$, being new field variables. Such an expansion is consistent with assumptions made in plate structure problems formulations when $h \ll L$. Then,

$$
\tau_{\alpha \beta}^{(\theta)}\left(\mathbf{x}, x_{3}\right)=\sum_{a=0}^{2} z^{a} \tau_{\alpha \beta}^{\left(\vartheta_{a}\right)}(\mathbf{x}), \tau_{\alpha \beta}^{\left(\vartheta_{a}\right)}(\mathbf{x}):=(1+v) \vartheta_{a}(\mathbf{x}) \delta_{\alpha \beta} \text { for }(\mathrm{a}=0,1,2)
$$

If we inserted (8) into the governing heat conduction equation, we would obtain the PDE

$$
\left[k_{H}(\mathbf{x}) k_{V}\left(x_{3}\right)\left(\sum_{a=0}^{2}\left(x_{3} / h\right)^{a} \vartheta_{a}(\mathbf{x})\right)_{, j}\right]_{, j}=0
$$

which is still dependent on $x_{3}$. Therefore, Eq. (10) will be considered in the averaged sense over the plate thickness. Introducing dimensionless coordinates and temperature fields

$$
x_{\beta}^{*}:=x_{\beta} / L, x_{3}^{*}:=x_{3} / h_{0}=h^{*}(\mathbf{x}) z, \vartheta_{a}^{*}(\mathbf{x}):=\vartheta_{a}(\mathbf{x}) / \theta_{0}
$$

the averaged heat conduction equation becomes

$$
\sum_{a=1}^{2} C^{\left(\theta \vartheta_{a}\right)}(\mathbf{x}) \vartheta_{a}^{*}(\mathbf{x})+\sum_{a=0}^{2} G_{\beta}^{\left(\theta \vartheta_{a}\right)}(\mathbf{x}) \vartheta_{a, \beta}^{*}(\mathbf{x})+\sum_{a=0}^{2} G^{\left(\theta \vartheta_{a}\right)}(\mathbf{x}) \vartheta_{a, \beta \beta}^{*}(\mathbf{x})=0,
$$

where

$$
\begin{gathered}
C^{\left(\theta \vartheta_{a}\right)}(\mathbf{x}):=\left(\frac{L}{h_{0}}\right)^{2} \frac{k_{H}(\mathbf{x})}{\left(h^{*}(\mathbf{x})\right)^{2}}\left[k_{V}(1 / 2)+(-1)^{a} k_{V}(-1 / 2)\right], a=1,2 \\
G_{\beta}^{\left(\theta \vartheta_{a}\right)}(\mathbf{x}):=\left(d_{(0) a}+\omega d_{(s) a}\right) k_{H, \beta}(\mathbf{x}), a=0,1,2 \\
G^{\left(\theta \vartheta_{a}\right)}(\mathbf{x}):=\left(d_{(0) a}+\omega d_{(s) a}\right) k_{H}(\mathbf{x}), a=0,1,2
\end{gathered}
$$


in which $d_{(0) a}:=\int_{-1 / 2}^{1 / 2} z^{a} d z=\frac{1+(-1)^{a}}{(a+1) 2^{a+1}}$

$$
d_{(p) a}:=\int_{-1 / 2}^{1 / 2} z^{a}\left(\frac{1}{2} \pm z\right)^{p} d z=( \pm 1)^{a} \int_{0}^{1}\left(y-\frac{1}{2}\right)^{a} y^{p} d y=( \pm 1)^{a} \sum_{k=0}^{a}\left(\begin{array}{l}
a \\
k
\end{array}\right)\left(-\frac{1}{2}\right)^{k} \frac{1}{p+1+a-k}
$$

Eq. (12) should be supplemented with thermal boundary conditions on the top and bottom surface of the plate. Usually, we distinguish three kinds of boundary conditions:

Dirichlet b.c.: $\vartheta_{0}^{*}(\mathbf{x}) \pm \frac{1}{2} \vartheta_{1}^{*}(\mathbf{x})+\frac{1}{4} \vartheta_{2}^{*}(\mathbf{x})=\frac{\bar{\theta}(\mathbf{x}, \pm 1 / 2)}{\theta_{0}}-1 ; \bar{\theta}(\mathbf{x}, \pm 1 / 2)$-prescribed

Neuman b.c.: $\pm k_{0} k_{H}(\mathbf{x}) k_{V}( \pm 1 / 2)\left[\vartheta_{1}^{*}(\mathbf{x}) \pm \vartheta_{2}^{*}(\mathbf{x})\right]=\frac{\bar{q}(\mathbf{x}, \pm 1 / 2)}{\theta_{0}}$;

$\bar{q}(\mathbf{x}, \pm 1 / 2)$ - prescribed heat fluxes

Robin b.c.: $\quad A\left[1+\vartheta_{0}^{*}(\mathbf{x}) \pm \frac{1}{2} \vartheta_{1}^{*}(\mathbf{x})+\frac{1}{4} \vartheta_{2}^{*}(\mathbf{x})\right] \pm B k_{0} k_{H}(\mathbf{x}) k_{V}( \pm 1 / 2)\left[\vartheta_{1}^{*}(\mathbf{x}) \pm \vartheta_{2}^{*}(\mathbf{x})\right]=0$.

In view of prescribed boundary conditions, one can eliminate $\vartheta_{1}$ and $\vartheta_{2}$ form (12) and $\vartheta_{0}^{*}(\mathbf{x})=\theta\left(\mathbf{x}, x_{3}=0\right) / \theta_{0}$ is the dimensionless temperature at the mid-plane of the plate. For $\vartheta_{0}^{*}$ , we need to prescribe also boundary conditions on the edge of the plate.

In order to proceed in the derivation of the variational formulation, we define certain semi-integral fields

$$
\begin{aligned}
& M_{\alpha \beta}^{(\varphi)}(\mathbf{x}):=c_{1} \int_{-h / 2}^{h / 2} \phi\left(x_{3}\right) \sigma_{\alpha \beta}\left(\mathbf{x}, x_{3}\right) d x_{3}, \\
& M_{\alpha \beta}^{(w)}(\mathbf{x}):=\int_{-h / 2}^{h / 2}\left(x_{3}-c_{1} \phi\left(x_{3}\right)\right) \sigma_{\alpha \beta}\left(\mathbf{x}, x_{3}\right) d x_{3}, \\
& T_{3 \beta}^{(w \varphi)}(\mathbf{x}):=c_{1}\left[\tilde{T}_{3 \beta}(\mathbf{x})-c_{2} Q_{3 \beta}(\mathbf{x})\right], \\
& T_{3 \beta}(\mathbf{x}):=\int_{-h / 2}^{h / 2} \sigma_{\alpha \beta}\left(\mathbf{x}, x_{3}\right) d x_{3}, Q_{3 \beta}(\mathbf{x}):=\int_{-h / 2}^{h / 2} \psi_{, 3}\left(x_{3}\right) \sigma_{\alpha \beta}\left(\mathbf{x}, x_{3}\right) d x_{3}
\end{aligned}
$$

where $\tilde{T}_{3 \beta}(\mathbf{x})$ is the Reissner modification of the shear stresses $T_{3 \beta}(\mathbf{x})$ by the shear correction factor as

$$
\tilde{T}_{3 \beta}(\mathbf{x}):=\left[\left(1-c_{2}\right) \kappa+c_{2}\right] T_{3 \beta}(\mathbf{x}) .
$$

Now, in view of (2)-(4), (7) and (15), (16), we may write

$$
\begin{aligned}
\delta U-\delta W= & -\int_{\Omega}\left\{T_{\alpha \beta, \beta}(\mathbf{x}) \delta u_{\alpha}(\mathbf{x})+\left[M_{\alpha \beta, \alpha \beta}^{(w)}(\mathbf{x})+T_{3 \beta, \beta}^{(w \varphi)}(\mathbf{x})+q(\mathbf{x})\right] \delta w(\mathbf{x})+\right. \\
& \left.+\left[M_{\alpha \beta, \beta}^{(\varphi)}(\mathbf{x})-T_{3 \alpha}^{(w \varphi)}(\mathbf{x})\right] \delta \varphi_{\alpha}(\mathbf{x})\right\} d \Omega+
\end{aligned}
$$




$$
\begin{aligned}
& +\int_{\partial \Omega}\left\{n_{\beta}(\mathbf{x}) T_{\alpha \beta}(\mathbf{x}) \delta u_{\alpha}(\mathbf{x})-n_{\alpha}(\mathbf{x}) n_{\beta}(\mathbf{x}) M_{\alpha \beta}^{(w)}(\mathbf{x}) \delta\left(\frac{\partial w}{\partial \mathbf{n}}(\mathbf{x})\right)+\right. \\
& \left.+n_{\beta}(\mathbf{x}) M_{\alpha \beta}^{(\varphi)}(\mathbf{x}) \delta \varphi_{\alpha}(\mathbf{x})+V(\mathbf{x}) \delta w(\mathbf{x})\right\} d \Gamma=0,
\end{aligned}
$$

where

$$
\begin{aligned}
& V(\mathbf{x}):=n_{\alpha}(\mathbf{x})\left(M_{\alpha \beta, \beta}^{(w)}(\mathbf{x})+T_{3 \alpha}^{(w \varphi)}(\mathbf{x})\right)+\frac{\partial}{\partial \mathbf{t}} T^{(w)}(\mathbf{x})-\sum_{c} \delta\left(\mathbf{x}-\mathbf{x}^{c}\right) \llbracket T^{(w)}\left(\mathbf{x}^{c}\right) \rrbracket \\
& T^{(w)}(\mathbf{x}):=t_{\alpha}(\mathbf{x}) n_{\beta}(\mathbf{x}) M_{\alpha \beta}^{(w)}(\mathbf{x})
\end{aligned}
$$

being the generalized shear force and the twisting moment defined on the plate edge. Furthermore, the jump at a corner on the oriented boundary edge $\partial \Omega$ is defined as

$$
\llbracket A\left(\mathbf{x}^{c}\right) \rrbracket:=A\left(\mathbf{x}^{c}+0\right)-A\left(\mathbf{x}^{c}-0\right) .
$$

Since $\left\{\delta u_{\alpha}, \delta w, \delta \varphi_{\alpha}\right\}$ are arbitrary in $\Omega$ and variation of particular fields are arbitrary on that part of the boundary where the field variable is not prescribed by the boundary conditions, one can deduce the governing equations and boundary conditions from the variational formulation Eq. (17). In the linear theory, it is convenient to utilize the formulation for dimensionless fields

$$
u_{\beta}^{*}(\mathbf{x}):=u_{\beta}(\mathbf{x}) / h_{0}, \varphi_{\beta}^{*}(\mathbf{x}, t):=\varphi_{\beta}(\mathbf{x}, t), w^{*}(\mathbf{x}):=w(\mathbf{x}) / h_{0} .
$$

Then, we obtain

governing equations:

$$
\begin{aligned}
& T_{\alpha \beta, \beta}^{*}(\mathbf{x})=0 \\
& M_{\alpha \beta, \alpha \beta}^{*(w)}(\mathbf{x})+\left(L / h_{0}\right)^{2} T_{3 \beta, \beta}^{*(w \varphi)}(\mathbf{x})=-p^{*}(\mathbf{x}) \\
& M_{\alpha \beta, \beta}^{*(\varphi)}(\mathbf{x})-\left(L / h_{0}\right)^{2} T_{3 \alpha}^{*(w \varphi)}(\mathbf{x})=0
\end{aligned}
$$

boundary conditions:

$$
\begin{aligned}
\left.u_{\alpha}^{*}(\mathbf{x})\right|_{\partial \Omega_{u}} \text { is prescribed and/or }\left.n_{\beta}(\mathbf{x}) T_{\alpha \beta}^{*}(\mathbf{x})\right|_{\partial \Omega_{t}} & =0 \\
\left.\frac{\partial w^{*}}{\partial \mathbf{n}}(\mathbf{x})\right|_{\partial \Omega_{w n}} \text { is prescribed and/or }\left.n_{\alpha}(\mathbf{x}) n_{\beta}(\mathbf{x}) M_{\alpha \beta}^{*(w)}(\mathbf{x})\right|_{\partial \Omega_{M}} & =0 \\
\left.\varphi_{\alpha}^{*}(\mathbf{x})\right|_{\partial \Omega_{\varphi}} \text { is prescribed and/or }\left.n_{\beta}(\mathbf{x}) M_{\alpha \beta}^{*(\varphi)}(\mathbf{x})\right|_{\partial \Omega_{M \varphi}} & =0 \\
\left.w^{*}(\mathbf{x})\right|_{\partial \Omega_{w}} \text { is prescribed and/or }\left.V^{*}(\mathbf{x})\right|_{\partial \Omega_{V}} & =0 .
\end{aligned}
$$

The explicit expressions for the semi-integral fields are given as

$$
\begin{aligned}
T_{\alpha \beta}^{*}(\mathbf{x})= & C^{(u u)}(\mathbf{x}) \tau_{\alpha \beta}^{*(u)}(\mathbf{x})+C^{(u \varphi)}(\mathbf{x}) \tau_{\alpha \beta}^{*(\varphi)}(\mathbf{x})+C^{(u w)}(\mathbf{x}) \tau_{\alpha \beta}^{*(w)}(\mathbf{x})+ \\
& +\sum_{a=0}^{2} C^{\left(u \vartheta_{a}\right)}(\mathbf{x}) \tau_{\alpha \beta}^{*\left(\vartheta_{a}\right)}(\mathbf{x})=\frac{L^{2}}{D_{0}} T_{\alpha \beta}(\mathbf{x})
\end{aligned}
$$




$$
\begin{aligned}
& C^{(u u)}(\mathbf{x}):=12 \frac{1-v}{H} \frac{L}{h_{0}}\left(d_{(0) 0}+\zeta d_{(p) 0}\right) D_{1 H}(\mathbf{x}) \\
& C^{(u \varphi)}(\mathbf{x}):=12 \frac{1-v}{H} \frac{L}{h_{0}} c_{1} \zeta\left(d_{(p) 1}-\frac{4}{3} c_{2} d_{(p) 3}\right) D_{2 H}(\mathbf{x}) \\
& C^{(u w)}(\mathbf{x}):=12 \frac{1-v}{H} \zeta\left[\left(c_{1}-1\right) d_{(p) 1}-\frac{4}{3} c_{1} c_{2} d_{(p) 3}\right] D_{2 H}(\mathbf{x}) \\
& C^{\left(u \vartheta_{a}\right)}(\mathbf{x}):=-12 \frac{1-v}{H}\left(\frac{L}{h_{0}}\right)^{2} \alpha_{0} \theta_{0}\left(d_{(0) a}+\xi d_{(r) a}+\zeta d_{(p) a}+\xi \zeta d_{(p+r) a}\right) B_{1 H}(\mathbf{x}) \\
& T_{3 \alpha}^{*(w \varphi)}(\mathbf{x}, t)=C^{s}(\mathbf{x})\left[w_{, \alpha}^{*}(\mathbf{x})+\frac{L}{h_{0}} \varphi_{\alpha}^{*}(\mathbf{x})\right]=\frac{L h_{0}}{D_{0}} T_{3 \alpha}(\mathbf{x}) \\
& C^{s}(\mathbf{x}):=c_{1} 6(1-v)\left\{\left[\left(1-c_{2}\right) \kappa+c_{2}\right]\left(d_{(0) 0}+\zeta d_{(p) 0}\right)-8 c_{2}\left(d_{(0) 2}+\zeta d_{(p) 2}\right)+\right. \\
& \left.+16 c_{2}\left(d_{(0) 4}+\zeta d_{(p) 4}\right)\right\} D_{1 H}(\mathbf{x}) \\
& M_{\alpha \beta}^{*(\varphi)}(\mathbf{x})=C^{(\varphi u)}(\mathbf{x}) \tau_{\alpha \beta}^{*(u)}(\mathbf{x})+C^{(\varphi \varphi)}(\mathbf{x}) \tau_{\alpha \beta}^{*(\varphi)}(\mathbf{x})+C^{(\varphi w)}(\mathbf{x}) \tau_{\alpha \beta}^{*(w)}(\mathbf{x})+ \\
& +\sum_{a=0}^{2} C^{\left(\varphi \vartheta_{a}\right)}(\mathbf{x}) \tau_{\alpha \beta}^{*\left(\vartheta_{a}\right)}(\mathbf{x})=\frac{L^{2}}{D_{0} h_{0}} M_{\alpha \beta}^{(\varphi)}(\mathbf{x}) \\
& C^{(\varphi u)}(\mathbf{x})=C^{(u \varphi)}(\mathbf{x}) \\
& C^{(\varphi \varphi)}(\mathbf{x}):=12 \frac{1-v}{H} \frac{L}{h_{0}} c_{1}\left[d_{(0) 2}-\frac{8}{3} c_{2} d_{(0) 4}+\frac{16}{9} c_{2} d_{(0) 6}+\right. \\
& \left.+\zeta\left(d_{(p) 2}-\frac{8}{3} c_{2} d_{(p) 4}+\frac{16}{9} c_{2} d_{(p) 6}\right)\right] D_{3 H}(\mathbf{x}) \\
& C^{(\varphi w)}(\mathbf{x}):=-16 \frac{1-v}{H} c_{1} c_{2}\left[d_{(0) 4}-\frac{4}{3} d_{(0) 6}+\zeta\left(d_{(p) 4}-\frac{4}{3} d_{(p) 6}\right)\right] D_{3 H}(\mathbf{x}) \\
& C^{\left(\varphi \vartheta_{a}\right)}(\mathbf{x}):=-12 \frac{1-v}{H}\left(\frac{L}{h_{0}}\right)^{2} \alpha_{0} \theta_{0} c_{1}\left[d_{(0) a+1}-\frac{4}{3} c_{2} d_{(0) a+3}+\xi\left(d_{(r) a+1}-\frac{4}{3} c_{2} d_{(r) a+3}\right)+\right. \\
& \left.+\zeta\left(d_{(p) a+1}-\frac{4}{3} c_{2} d_{(p) a+3}\right)+\xi \zeta\left(d_{(p+r) a+1}-\frac{4}{3} c_{2} d_{(p+r) a+3}\right)\right] B_{2 H}(\mathbf{x}) \\
& M_{\alpha \beta}^{*(w)}(\mathbf{x})=C^{(w u)}(\mathbf{x}) \tau_{\alpha \beta}^{*(u)}(\mathbf{x})+C^{(w \varphi)}(\mathbf{x}) \tau_{\alpha \beta}^{*(\varphi)}(\mathbf{x})+ \\
& +C^{(w w)}(\mathbf{x}) \tau_{\alpha \beta}^{*(w)}(\mathbf{x})+\sum_{a=0}^{2} C^{\left(w \vartheta_{a}\right)}(\mathbf{x}) \tau_{\alpha \beta}^{*\left(\vartheta_{a}\right)}(\mathbf{x})=\frac{L^{2}}{D_{0} h_{0}} M_{\alpha \beta}^{(w)}(\mathbf{x})
\end{aligned}
$$




$$
\begin{aligned}
C^{(w u)}(\mathbf{x}):= & 12 \frac{1-v}{H} \frac{L}{h_{0}} \zeta\left[\left(1-c_{1}\right) d_{(p) 1}+\frac{4}{3} c_{1} c_{2} d_{(p) 3}\right] D_{2 H}(\mathbf{x})=-\frac{L}{h_{0}} C^{(u w)}(\mathbf{x}) \\
C^{(w \varphi)}(\mathbf{x}):= & 16 \frac{1-v}{H} \frac{L}{h_{0}} c_{1} c_{2}\left[d_{(0) 4}-\frac{4}{3} d_{(0) 6}+\zeta\left(d_{(p) 4}-\frac{4}{3} d_{(p) 6}\right)\right] D_{3 H}(\mathbf{x})=-\frac{L}{h_{0}} C^{(\varphi w)}(\mathbf{x}) \\
C^{(w w)}(\mathbf{x}):= & 12 \frac{1-v}{H}\left\{\left(c_{1}-1\right) d_{(0) 2}-\frac{16}{9} c_{1} c_{2} d_{(0) 6}+\right. \\
& \left.+\zeta\left[\left(c_{1}-1\right) d_{(p) 2}-\frac{16}{9} c_{1} c_{2} d_{(p) 6}\right]\right\} D_{3 H}(\mathbf{x}) \\
C^{\left(w \vartheta_{a}\right)}(\mathbf{x}):= & 12 \frac{1-v}{H}\left(\frac{L}{h_{0}}\right)^{2} \alpha_{0} \theta_{0}\left\{\left(c_{1}-1\right) d_{(0) a+1}-\frac{4}{3} c_{1} c_{2} d_{(0) a+3}+\right. \\
& +\xi\left[\left(c_{1}-1\right) d_{(r) a+1}-\frac{4}{3} c_{1} c_{2} d_{(r) a+3}\right]+\zeta\left[\left(c_{1}-1\right) d_{(p) a+1}-\frac{4}{3} c_{1} c_{2} d_{(p) a+3}\right]+ \\
& \left.+\xi \zeta\left[\left(c_{1}-1\right) d_{(p+r) a+1}-\frac{4}{3} c_{1} c_{2} d_{(p+r) a+3}\right]\right\} B_{2 H}(\mathbf{x}),
\end{aligned}
$$

where

$$
\begin{aligned}
& \tau_{\alpha \beta}^{*(u)}(\mathbf{x})=\frac{H}{2}\left(u_{\alpha, \beta}^{*}(\mathbf{x})+u_{\beta, \alpha}^{*}(\mathbf{x})\right)+v \delta_{\alpha \beta} u_{\gamma, \gamma}^{*}(\mathbf{x})=\frac{L}{h_{0}} \tau_{\alpha \beta}^{(u)} \\
& \tau_{\alpha \beta}^{*(\varphi)}(\mathbf{x})=\frac{H}{2}\left(\varphi_{\alpha, \beta}^{*}(\mathbf{x})+\varphi_{\beta, \alpha}^{*}(\mathbf{x})\right)+v \delta_{\alpha \beta} \varphi_{\gamma, \gamma}^{*}(\mathbf{x})=L \tau_{\alpha \beta}^{(\varphi)} \\
& \tau_{\alpha \beta}^{*(w)}(\mathbf{x})=H w_{, \alpha \beta}^{*}(\mathbf{x})+v \delta_{\alpha \beta} \nabla^{2} w^{*}(\mathbf{x})=\frac{L^{2}}{h_{0}} \tau_{\alpha \beta}^{(w)} \\
& \tau_{\alpha \beta}^{*\left(\vartheta_{a}\right)}(\mathbf{x})=(1+v) \vartheta_{a}^{*}(\mathbf{x}) \delta_{\alpha \beta}=\tau_{\alpha \beta}^{\left(\vartheta_{a}\right)} / \theta_{0} \\
& D_{j H}(\mathbf{x}):=\left(h^{*}(\mathbf{x})\right)^{j} E_{H}(\mathbf{x}), B_{j H}(\mathbf{x}):=\left(h^{*}(\mathbf{x})\right)^{j} E_{H}(\mathbf{x}) \alpha_{H}(\mathbf{x}), \\
& D_{0}=\frac{E_{0}\left(h_{0}\right)^{3}}{12\left(1-v^{2}\right)}, p^{*}(\mathbf{x}):=\frac{L^{4}}{D_{0} h_{0}} p(\mathbf{x}) .
\end{aligned}
$$

\section{NUMERICAL IMPLEMENTATION}

The MLS-approximation belongs to mesh-free approximations, since no predefined connectivity among nodal points is required [6]. The nodal points are freely distributed inside the analyzed domain and on its boundary. Beside the standard MLS-approximation, one can utilize also the Central Approximation Node (CAN) concept [7, 8] which enables to utilize the local support character of the approximation efficiently. In the case of strong formulations, however, the advantages of the local support approximation are utilized immediately 
$[9,10]$. The MLS-approximation of a field variable $g(\mathbf{x})$ is expressed in terms of the shape functions $\psi^{\bar{a}}(\mathbf{x})$ and certain nodal unknowns $\hat{g}^{\bar{a}}$ as

$$
\left.g(\mathbf{x})\right|_{\Omega^{q}}=\sum_{a=1}^{N^{q}} \hat{g}^{\bar{a}} \psi^{\bar{a}}(\mathbf{x}), \Omega \supset \Omega^{q} \ni \mathbf{x}^{q},
$$

where the central approximation nodal point $\mathbf{x}^{q}$ can be selected as the nearest nodal point to the point of approximation $\mathbf{x}$, and $\bar{a}:=n(q, a)$ is the global number while $a$ is the local number of the node from the support domain of the nodal point $\mathbf{x}^{q}$.

In order to eliminate the $3 \mathrm{rd}$ and 4 th order derivatives of field variables in the governing equations, we propose to utilize the decomposition of the derived system of the PDE into a set of PDE with derivatives not higher than second order [3] by introducing new field variables

$$
m^{*}(\mathbf{x}):=\nabla^{2} w^{*}(\mathbf{x}), s_{\alpha}^{*}(\mathbf{x}):=\nabla^{2} u_{\alpha}^{*}(\mathbf{x}), f_{\alpha}^{*}(\mathbf{x}):=\nabla^{2} \varphi_{\alpha}^{*}(\mathbf{x}) \text { in } \Omega \cup \partial \Omega
$$

\section{NUMERICAL EXAMPLES}

Let us consider the square plate $L \times L(L=1)$ with clamped edges and constant thickness $h=h_{0}=L / 50$. The plates are either homogeneous or FGM with power-law gradation of material coefficients according to (6) and constant Poisson ratio $v=0.3$. In presentation of numerical results, we focus on the study of the deflection response to thermal loading with prescribed temperatures on the bottom and top of the plate, $\theta^{*}(\mathbf{x},-h / 2)=0, \theta^{*}(\mathbf{x}, h / 2)=20$, and vanishing heat flux on the lateral sides $\left.k n_{\alpha}(\mathbf{x}) \vartheta_{0, \alpha}^{*}(\mathbf{x})\right|_{\partial 0}=0$.

The first conclusion is that there is no deflection response to the prescribed thermal loading neither in the homogeneous nor in FGM plates even if any one of the coefficients $\{E, \alpha, k\}$ is graded non-linearly in the transversal direction. Neither the combination of gradations of material coefficients in transversal direction gives rise to finite deflections in both the KLT and TSDPT.

Another observation is that the deflections are independent on the heat conduction coefficient gradation.

It can be seen from the analysis of governing equations that there is a coupling between deflections and thermal fields in the KLT only if $\nabla^{2}\left(E_{H}(\mathbf{x}) \alpha_{H}(\mathbf{x})\right) \neq 0$. The numerical results (Fig. 1) confirm this finding. Really, finite deflections appeared when at least one of the coefficients $\left(E_{H}(\mathbf{x}), \alpha_{H}(\mathbf{x})\right)$ was graded non-linearly, or both of them were graded at least linearly.

In contrast to the KLT, finite deflections occur in the TSDPT even if $\nabla\left(E_{H}(\mathbf{x}) \alpha_{H}(\mathbf{x})\right) \neq 0$ because of coupling between rotations $\varphi_{\alpha}$ and temperature and another coupling between deflections and rotations in TSDPT. This indirect coupling between deflections and temperature is significantly weaker than the direct $\left(w-\vartheta_{0}\right)$-coupling occurring in FGM plates with such an in-plane gradation of material coefficients that $\nabla^{2}\left(E_{H}(\mathbf{x}) \alpha_{H}(\mathbf{x})\right) \neq 0$ (see Fig. 2).

The additional transversal gradation of the YM and/or linear heat extension coefficient has an influence on finite deflections in FGM plates with combined in-plane gradations of $E_{H}(\mathbf{x})$ and $\alpha_{H}(\mathbf{x})$. The additional transversal gradation of the heat extension coefficient increases the deflections in both the KLT and TSDPT, while the gradation of the YM has an opposite effect on deflections. Note that in the case of TSDPT, the deflections change the sign when the transversal gradation of YM is applied additionally (Fig. 3). 

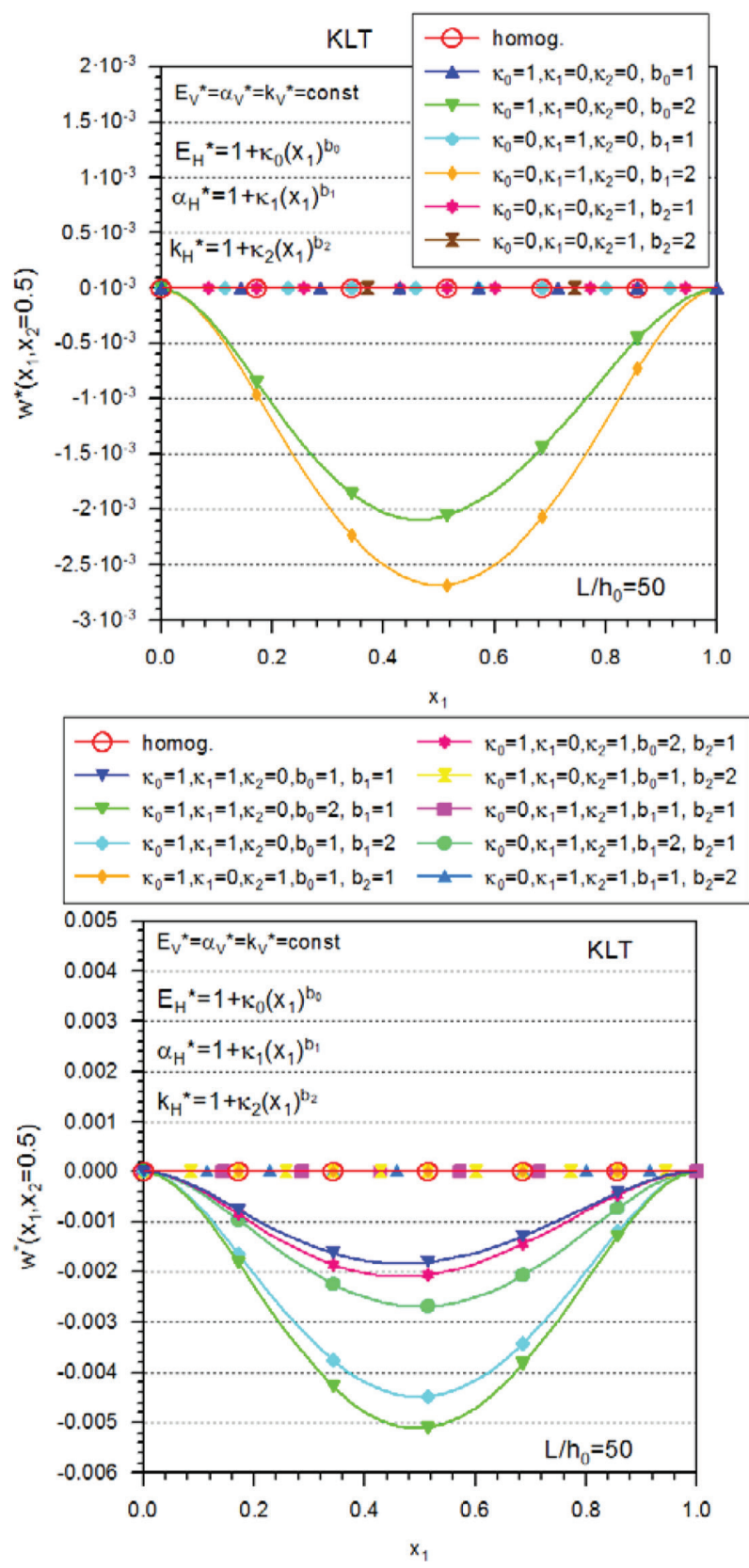

Figure 1: Deflections in FGM plates with in-plane gradation of material coefficients; results by KLT. 


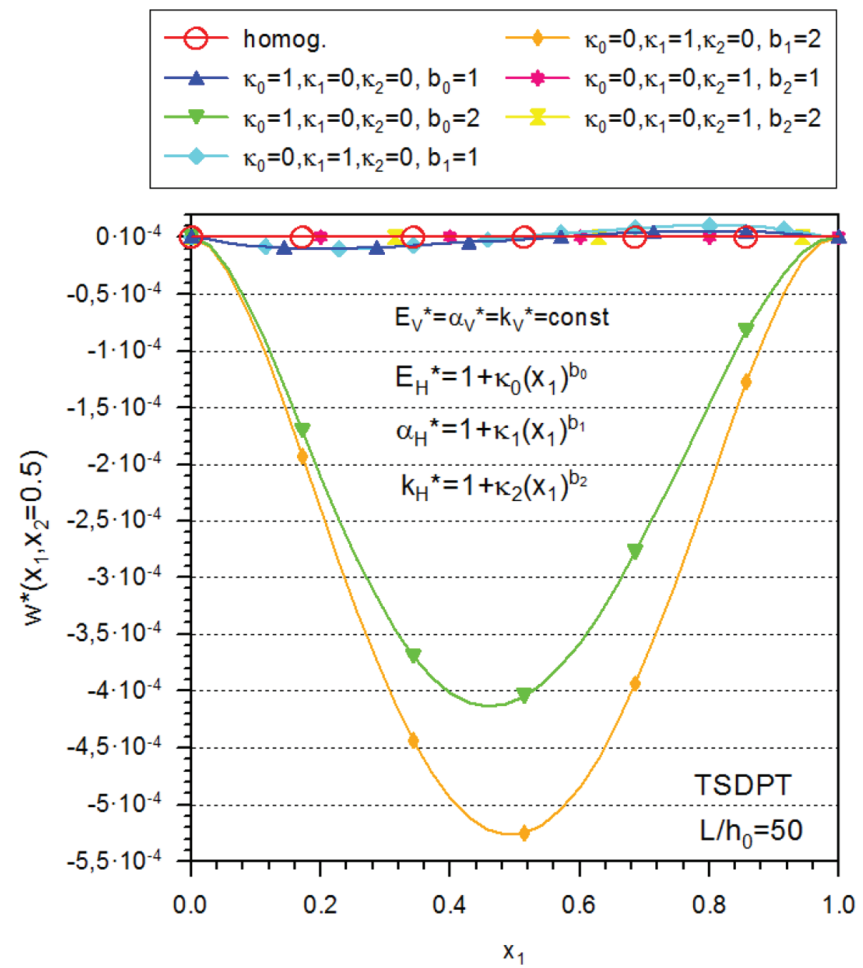

Figure 2: Deflections in FGM plates with in-plane gradation of material coefficients; results by TSDPT.

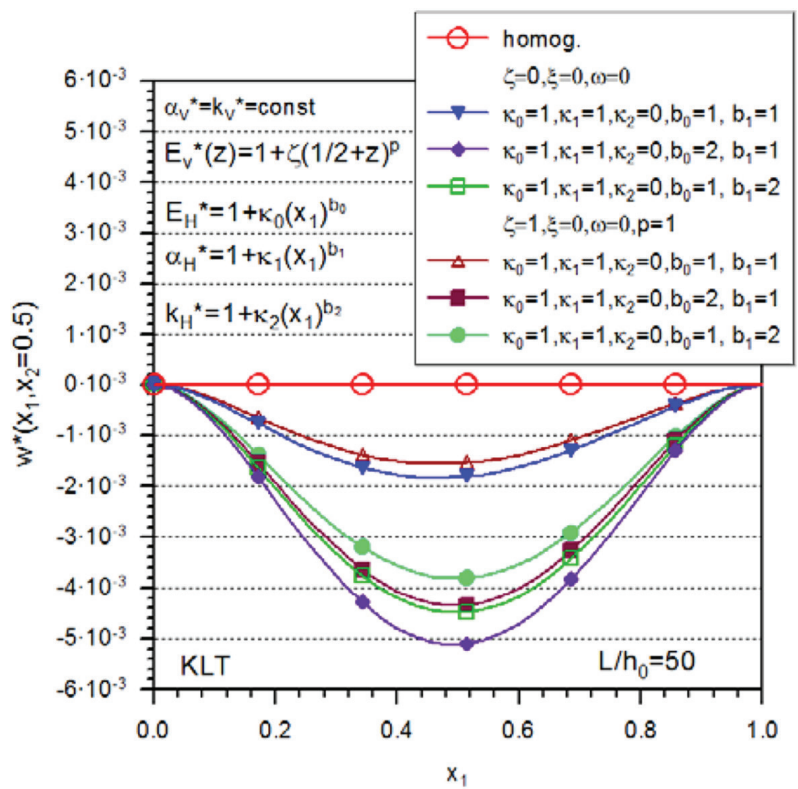

Figure 3: Deflections in FGM plates with combined in-plane gradation of material coefficients and transversal gradation of YM; results by KLT and TSDPT. 


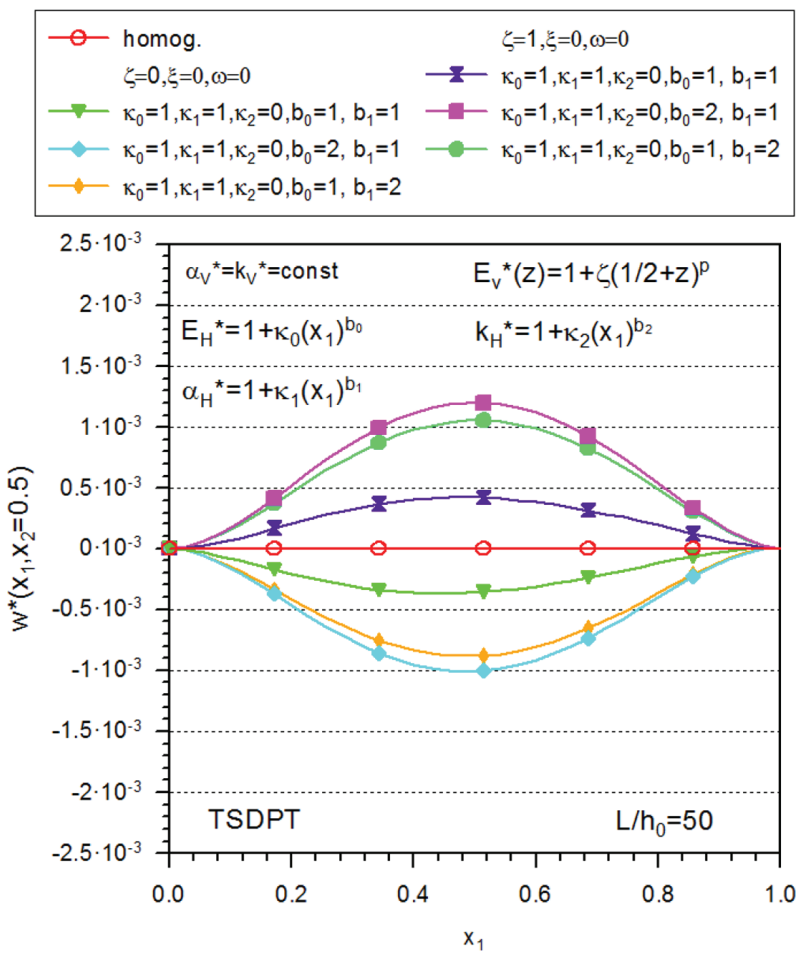

Figure 3: (Continued)

\section{ACKNOWLEDGEMENTS}

This work was partially supported by the Slovak Research and Development Agency under the contract No. APVV-14-0440.

\section{REFERENCES}

[1] Birman, V. \& Byrd, L.W., Modeling and analysis of functionally graded materials and structures. Applied Mechanics Reviews, 60, pp. 195-216, 2007. https://doi.org/10.1115/1.2777164

[2] Daniel, I.M. \& Ishai, O., Engineering Mechanics of Composite Materials. Oxford University Press: New York, 2006.

[3] Sladek, V., Sladek, J. \& Sator, L., Physical decomposition of thin plate bending problems and their solution by mesh-free methods. Engineering Analysis with Boundary Elements, 37, pp. 348-365, 2013. https://doi.org/10.1016/j.enganabound.2012.11.005

[4] Sator, L., Sladek V. \& Sladek J., Coupling effects in elastic analysis of FGM composite plates by mesh-free methods. Composite Structures, 115, pp. 100-110, 2014. https://doi.org/10.1016/j.compstruct.2014.04.016

[5] Sator, L., Sladek, V. \& Sladek, J., Multi-gradation coupling effects in FGM plates. Composite Structures, 171, pp. 515-527, 2017. https://doi.org/10.1016/j.compstruct.2017.03.063

[6] Lancaster, P. \& Salkauskas, K., Surfaces generated by moving least square method. Mathematics of Computation, 37, pp. 141-158, 1981. https://doi.org/10.1090/s0025-5718-1981-0616367-1 
[7] Sladek, V., Sladek, J. \& Zhang, Ch., Computation of stresses in non-homogeneous elastic solids by local integral equation method: a comparative study. Computational Mechanics, 41, pp. 827-845, 2008. https://doi.org/10.1007/s00466-006-0155-5

[8] Sladek V., Sladek J. \& Zhang Ch., Local integral equation formulation for axially symmetric problems involving elastic FGM. Engineering Analysis with Boundary Elements, 32, pp. 1012-1024, 2008.

https://doi.org/10.1016/j.enganabound.2007.09.006

[9] Sladek V., Sladek J. \& Zhang Ch., On increasing computational efficiency of local integral equation method combined with meshless implementations. CMES-Computer Modeling in Engineering \& Sciences, 63, pp. 243-263, 2010.

[10] Sladek, V. \& Sladek, J., Local integral equations implemented by MLS-approximation and analytical integrations. Engineering Analysis with Boundary Elements, 34, pp. 904-913, 2010.

https://doi.org/10.1016/j.enganabound.2010.03.015 